\section{Out-of-hospital cardiac arrest: incidence, process of care, and outcomes in an urban city, Korea}

\author{
Hanjin Cho', Sungwoo Moon ${ }^{1}$, Sung Joon Park', Gapsu Han', \\ Jong-Hak Park ${ }^{1}$, Hongjae Lee ${ }^{2}$, Jungoh $\mathrm{Choi}^{3}$, Seungsik Hwang ${ }^{4}$, \\ Bentley J Bobrow ${ }^{5-7}$
}

\author{
'Department of Emergency Medicine, Korea University Ansan Hospital, Ansan, Korea \\ ${ }^{2}$ Ansan Public Health Center, Ansan, Korea \\ ${ }^{3}$ Ansan Fire Department, Ansan, Korea \\ ${ }^{4}$ Department of Social and Preventive Medicine, Inha University School of Medicine, Incheon, Korea \\ ${ }^{5}$ Arizona Emergency Medicine Research Center, University of Arizona College of Medicine, Phoenix, AZ, USA \\ ${ }^{6}$ Department of Emergency Medicine, Maricopa Medical Center, Phoenix, AZ, USA \\ ${ }^{7}$ Arizona Department of Health Services, Bureau of EMS and Trauma Systems, Phoenix, AZ, USA
}

Objective We aimed to determine the incidence, processes of care, and outcomes in out-of-hospital cardiac arrests (OHCA) in Ansan, South Korea.

Methods From the Ansan Fire Department's (1-1-9 emergency call number) Emergency Medical Services (EMS) database, we obtained a list of adult cardiac arrest cases occurring between January 2008 and December 2011. We excluded cases with obvious non-cardiac causes, such as trauma, drowning, hanging, and asphyxia. We matched the EMS data with in-hospital care and outcome data. We analyzed basic demographic variables (age and gender), the time and place of incidence, witnesses, bystander cardiopulmonary resuscitation (CPR), major time variables, CPR instructions during transport, initial cardiac rhythm at the scene, and automated defibrillator use.

Results The overall incidence of OHCA in Ansan was 33.1/100,000 persons per year. Out of 778 adult OHCAs in our study population, bystander CPR was provided in 103 cases (13.2\%). Of the 517 OHCAs whose initial rhythms were confirmed, 85 (16.4\%) showed shockable rhythms, but only $23(27.1 \%)$ received defibrillation at the scene or during transportation. Of the 106 patients whose spontaneous circulation returned at the hospital, only 6 (5.7\%) received mild therapeutic hypothermia. During the study period, 31 patients (4\%) survived to discharge from hospitals, and 6 of these discharged patients (19.4\%) showed favorable neurologic outcomes.

Conclusion While the survival rate from OHCA in Ansan is very low, this study provides basic information needed to create improvements. Our analysis suggests that multiple variables contribute to the low OHCA survival rate. Several of these variables are modifiable; addressing them is a clear first step toward strengthening the chain of survival from OCHA in Ansan.

Keywords Out-of-hospital cardiac arrest; Resuscitation; Patient outcome assessment; Incidence; Urban health; Korea
elSSN: $2383-4625$

Received: 12 July 2014

Revised: 12 August 2014

Accepted: 12 August 2014

Correspondence to: Sungwoo Moon Department of Emergency Medicine, Korea University Ansan Hospital, 123 Jeokgeum-ro, Danwon-gu, Ansan 425-707, Korea

E-mail: sungwoo.moon89@gmail.com

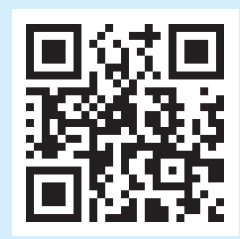

How to cite this article:

Cho H, Moon S, Park SJ, Han G, Park JH, Lee $H_{1}$ Choi J, Hwang S, Bobrow BJ. Out-ofhospital cardiac arrest: incidence, process of care, and outcomes in an urban city, Korea. Clin Exp Emerg Med 2014;1(2):94-100.

This is an Open Access article distributed under the terms of the Creative Commons Attribution Non-Commercial License (http:// creativecommons.org/licenses/by-nc/3.0/). 


Capsule
$\begin{aligned} & \text { What is already known } \\ & \text { The survival rate from out of hospital cardiac arrest in Korea is still low compared with other developed countries. } \\ & \text { Moreover, the survival rates in small cities near the capital are much lower than that of Seoul metropolitan area. }\end{aligned}$
$\begin{aligned} & \text { What is new in the current study } \\ & \text { To improve survival rate from out-of-hospital cardiac arrests (OHCA), multiple variables should be modified, according } \\ & \text { to the local features. This study is the first step necessary in improving survival within small cities in Gyeonggi-do prov- } \\ & \text { ince, Korea. }\end{aligned}$

\section{INTRODUCTION}

Out-of-hospital cardiac arrest (OHCA) is one of the leading causes of death, and it is regarded as an important public health issue. ${ }^{1-3}$ Developed countries have established community response systems for OHCA, achieving remarkable improvement, but $\mathrm{OHCA}$ outcomes still vary greatly among cities and countries. ${ }^{1,4}$

South Korea has recently shown increased interest in OHCA, making efforts to improve its survival rate. For example, the Korean Center for Disease Control and Prevention launched a Cardiovascular Disease Surveillance project to collect OHCA data in South Korea. ${ }^{5}$ While this database provides a national picture of the state of $\mathrm{OHCA}$, the information it contains is insufficient for a detailed understanding of OHCA in specific communities.

Effective responses to OHCA require emergency medical services (EMS), hospitals, and the public to integrate care and continually monitor interventions. ${ }^{6,7}$ To work toward this ideal response, healthcare organizations must first understand the current state of OHCA treatment in their community. This study aimed to assess the state of OHCA treatment in Ansan City by analyzing the OHCA incidence, processes of care, and outcomes in Ansan City. Our analysis of this information provides a baseline from which this community can work toward improved OHCA outcomes. To our knowledge, this is the first study in South Korea to describe in detail a regional community's response to OHCA.

\section{METHODS}

\section{Study setting}

Ansan City is approximately a one-hour drive from Seoul Metropolitan City and has an area of approximately $150 \mathrm{~km}^{2}$. It consists of residential, commercial, agricultural, and large industrial complexes. As of 2012, the population of Ansan was 759,402 (391,819 men and 367,583 women). ${ }^{8}$ Prehospital EMS in Ansan City are provided by the Fire Department (1-1-9 emergency call number), which is a governmental organization. The Ansan Fire Depart- ment has 10 ambulance stations located throughout the city. The department currently employs 23 level I and 25 level II EMS providers and two short-term trained emergency medical technicians. Only level I EMS providers may perform advanced airway management procedures (orotracheal intubation and supraglottic airway insertion) and initiate peripheral intravenous access (under the supervision of medical directors). EMS providers are trained to perform cardiopulmonary resuscitation (CPR) in all cases of cardiac arrest, unless there is evidence of irreversible death (rigor mortis or decapitation) or a 'Do not resuscitate' card is present.

\section{Study design and participants}

This was a retrospective cohort review of adult (18 years or older) OHCA in which cardiac arrest was recognized by EMS providers of the Ansan Fire Department (excluding cases in which arrest occurred after EMS arrival). Using the Ansan Fire Department EMS database, we obtained a list of adult cardiac arrest cases between January 2008 and December 2011. We excluded from the sample all cases with obvious non-cardiac causes, such as trauma, drowning, hanging, and asphyxia.

\section{Data collection and processing}

The importance of OHCA to public health prompted Ansan City officials to form the "Committee for Development in Emergency Medical Responses in Ansan City." The committee members were directors and managers of the city's Public Health Center, representing the Fire Department's EMS providers and physicians from the Emergency Medical Centers.

Our team of two trained researchers (one nurse and one emergency physician) used the EMS database of the Ansan Fire Department to gather information about OHCA response in Ansan City. We gathered information on basic demographics (age and gender), the time and place of incidence, witnesses, bystander CPR, CPR instructions during transport, initial cardiac rhythm at the scene, use of an automated defibrillator and major time variables: time between emergency call and EMS arrival, EMS time 
spent at the scene, and transport time to the hospital.

We also examined hospital data on our sample. There are 12 hospitals in Ansan City to which cardiac arrest patients are transported ( $A$ through $L$ ). $A$ is a regional emergency medical center with approximately 400 beds, $B$ is a regional emergency medicine clinic with approximately 300 beds, and $C$ is a university hospital that functions as a regional emergency medical center with approximately 600 beds. D through $L$ are hospitals of about 100 beds. We were able to match charts from these hospitals with the corresponding emergency medical transportation (EMT) data by comparing patients' names (when available on EMT charts), ages, and times of transport to the hospital.

Hospital medical records were obtained in two steps: (1) hospitals received a patient list and a form to fill out and (2) the trained investigators visited the hospitals to verify the data and collect any missing information. Through the medical records investigation, we obtained information on the recovery of spontaneous circulation (ROSC), survival and postcardiac arrest care, including whether therapeutic hypothermia $(\mathrm{TH})$ was delivered and whether coronary angiography was performed. The primary outcome we examined was survival to discharge, measured using the cerebral performance category (CPC) score in patients' medical records at the time of discharge or transfer. The report forms and data elements in this study were based on the Utstein style reporting template. $^{9}$

\section{Statistical analysis}

Prehospital and hospital data were entered into a Microsoft Excel (Microsoft Co., Redmond, VA, USA) spreadsheet, then transferred to the Stata 12.1 (StataCorp, College Station, TX, USA) program for statistical analysis. Normally distributed continuous data are reported as a mean \pm standard deviation (SD), and non-normally distributed continuous data are reported as a median with an accompanying interquartile range (IQR). A chi-square test was used to compare categorical variables. A multivariable model analyzed age, sex, witness status, public location, bystander CPR, EMS response interval, and initial EMS rhythm. The decision to include these covariates was based on the scientific understanding that these characteristics can influence resuscitation prognosis. Hosmer and Lemeshow tests were used to evaluate modeling fitness. A multivariate logistic regression analysis was performed to identify factors that significantly influenced survival outcomes. We calculated odds ratios (OR) to determine the strength of these associations, using 95\% confidence intervals.

\section{Ethics statement}

This study was approved by the Institutional Review Board of Ko- rea University Ansan Hospital (IRB number: AS 12014). Under the board's approval, we were exempted from obtaining written informed consent from enrolled patients.

\section{RESULTS}

During the study period, the total number of EMS-assessed OHCA patients was 1,218, and resuscitation was attempted in 1,083 cases. After excluding cases involving trauma, drowning, asphyx$\mathrm{ia}$, and arrests that occurred after EMS arrival, we analyzed 778 adult OHCA cases. The adjusted incidence of OHCA was approximately $33.1 / 100,000$ persons per year. The mean age of the patients was $64.9 \pm 18.2$ years. There were 458 male patients (58.9\%) and 320 female patients (41.1\%) (Table 1). The core data elements of OHCAs in Ansan City are displayed in Fig. 1 according to the Utstein reporting template.

Overall, 31/778 patients (4\%) survived to discharge during the study period. Neurological outcomes were confirmed in 21/31 (67.7\%) discharged patients: 6/31 (19.4\%) had a favorable neurological outcome (CPC score of 1 or 2 ) and 12/31 (38.7\%) had a poor neurological outcome (CPC score of 4).

\section{Community-level variables}

Of the 778 cases, 295 (37.9\%) were witnessed. Bystander CPR was provided in 103 cases (13.2\%). No cases of automated external defibrillation were reported. Location information indicated that $511 / 778$ of these cardiac arrests (65.7\%) occurred in residential areas, and the remaining 267/778 (34.3\%) occurred in public places (Fig. 1).

Table 1. Characteristics of included out-of-hospital cardiac arrest cases $(n=778)$

\begin{tabular}{lc}
\hline Characteristic & No. of patients (\%) \\
\hline Gender & \\
Male & $458(58.9)$ \\
Female & $320(41.1)$ \\
Age (yr), mean \pm SD & $64.9 \pm 18.2$ \\
Location & \\
Private home & $511(65.7)$ \\
Public & $200(25.7)$ \\
Unknown & $67(8.6)$ \\
Witnessed by bystander & $295(37.9)$ \\
Resuscitation attempted by bystander & $103(13.2)$ \\
AED application by bystander & 0 \\
Initial rhythm at the scene ${ }^{a)}$ & \\
Shockable & $85(16.4)$ \\
Not shockable & $432(83.6)$ \\
\hline
\end{tabular}

a)This does not include the 261 patients whose initial rhythms were not recorded at the scene.

$A E D$, automated external defibrillator. 


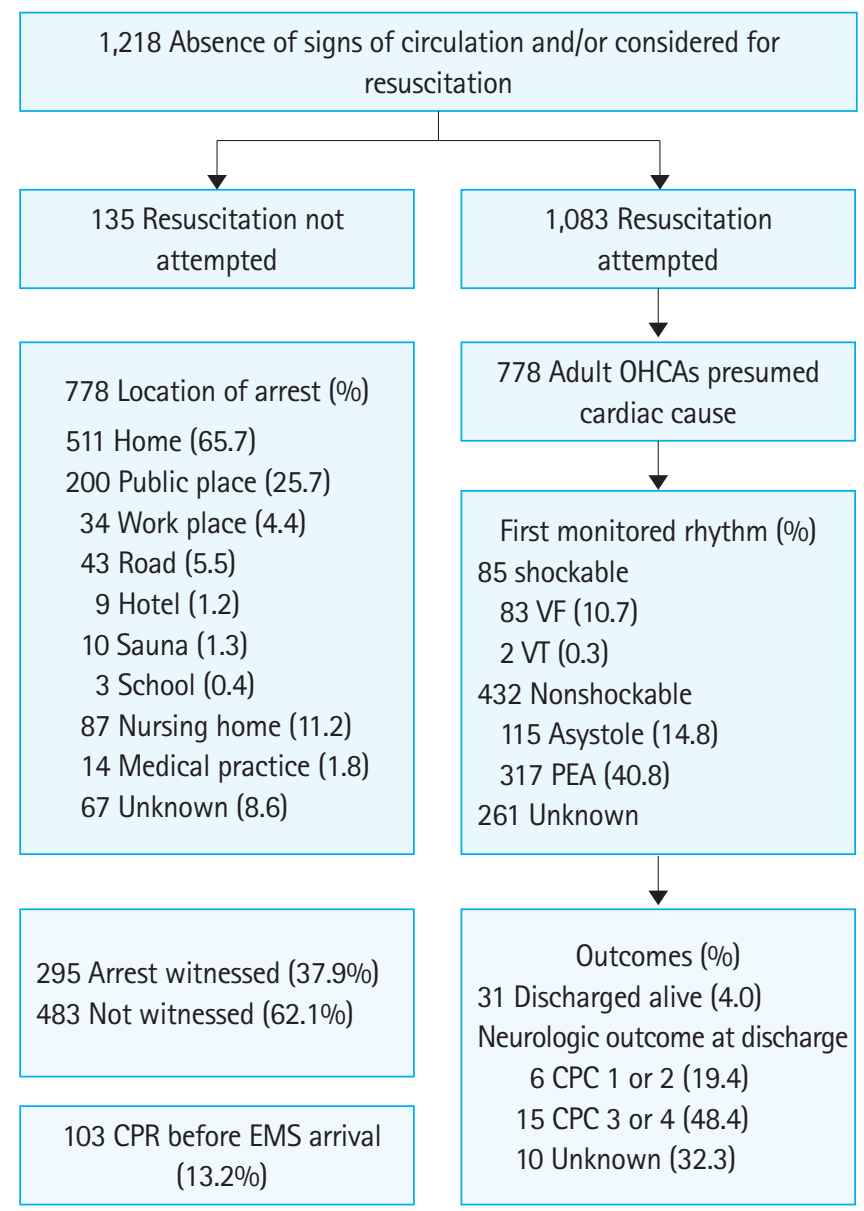

Fig. 1. The core data elements of out-of-hospital cardiac arrests (OHCAs) in Ansan City according to the Utstein reporting template.

$\mathrm{VF}$, ventricular fibrillation; $\mathrm{VT}$, ventricular tachycardia; PEA, pulseless electrical activity; EMS, emergency medical service; $C P C$, cerebral performance category.

\section{EMS-level variables}

The median response time between the report of the incident and the arrival of EMS providers was 6 minutes (IQR, 5 to 9 minutes). EMS providers spent a median of 5 minutes (IQR, 3 to 8 minutes) at the scene to resuscitate and prepare patients for transport. The median transportation distance to the hospital was $2 \mathrm{~km}(\mathrm{IQR}, 1.5$ to $3.5 \mathrm{~km}$ ), and the median transportation time was 5 minutes (IQR, 3 to 6 minutes).

Of the 778 OHCAs, the initial cardiac rhythm at the scene was confirmed in 517/778 (66.5\%) cases. Among these, 85/517 (16.4\%) showed shockable rhythms, 115/517 (22.3\%) showed pulseless electrical activity and 317/517 (61.3\%) showed asystole. Of the 83 cases of ventricular fibrillation and the two cases of ventricular tachycardia that required defibrillation, only 23 patients $(27.1 \%)$ received defibrillation by EMS providers prior to arrival at the hospital.
Table 2. Prearrival CPR instructions by emergency medical service providers and provision of bystander CPR

\begin{tabular}{lccrl}
\hline \multirow{2}{*}{$\begin{array}{l}\text { Prearrival CPR } \\
\text { instruction }\end{array}$} & \multicolumn{3}{c}{ Bystander CPR provision } & \multirow{2}{*}{ P-value } \\
\cline { 2 - 4 } & Total & Yes & No & \\
\hline Yes & 111 & $57(51.4)$ & $54(48.7)$ & $<0.001$ \\
No & 667 & $46(6.9)$ & $621(93.1)$ & \\
Total & 778 & $103(13.2)$ & $675(86.8)$ & \\
\hline
\end{tabular}

Values are presented as number (\%).

CPR, cardiopulmonary resuscitation.

Table 3. Number of transferred out-of-hospital cardiac arrest (OHCA) patients and survival rate by hospital

\begin{tabular}{lcc}
\hline Hospital $^{\text {a) }}$ & Transferred patients & Discharged alive \\
\hline A & 191 & $8(4.2)$ \\
B & 171 & $3(1.8)$ \\
C & 148 & $9(6.1)$ \\
D & 61 & $2(3.3)$ \\
E & 59 & $2(3.4)$ \\
F & 42 & 0 \\
G & 34 & $2(5.9)$ \\
H & 28 & $1(3.4)$ \\
I & 22 & $2(9.1)$ \\
J & 11 & $1(9.1)$ \\
K & 6 & $1(16.7)$ \\
L & 5 & 0 \\
Total & 778 & $31(4)$ \\
\hline
\end{tabular}

Values are presented as number (\%).

a) Listed as the order of the number of transferred OHCA patients. A is a regional emergency medical center with approximately 400 beds. $B$ is a regional emergency medicine clinic with approximately 300 beds. $C$ is a teaching tertiary university hospital that functions as a regional emergency medical center with approximately 600 beds. The hospitals $D$ through $L$ are hospitals containing about 100 beds.

In 111/778 cases (14.3\%), 1-1-9 prearrival telephone CPR instructions were given to bystanders, and 57/111 patients (51.4\%) received bystander CPR (Table 2). The probability of bystander CPR was significantly higher for cases in which prearrival CPR instructions were provided to the lay rescuers.

\section{Hospital-level variables}

Patients were transported to one of 12 hospitals in the Ansan City area. The number of patients and their survival rates by hospital are shown in Table 3. Table 4 shows the results of integrated postresuscitation care and the survival rates in the 3 hospitals that treated 100 or more OHCA patients during the study period. Of the 106 ROSC patients, only 6 (5.7\%) received mild therapeutic hypothermia. Survival rates differed among hospitals, but these differences were not statistically significant. 
Table 4. Postresuscitation care and survival rates in the major transfer hospitals

\begin{tabular}{lccccc} 
Hospital & $\begin{array}{c}\text { No. of } \\
\text { patients }\end{array}$ & ROSC & PCl & MTH & $\begin{array}{c}\text { Discharged } \\
\text { alive }^{\text {b) }}\end{array}$ \\
\hline A & 148 & $26(17.6)$ & $8(5.4)$ & $5(3.4)$ & $9(6.1)$ \\
B & 191 & $51(26.7)$ & $3(1.6)$ & 0 & $8(4.2)$ \\
C & 171 & $29(17.0)$ & $2(1.2)$ & $1(0.6)$ & $3(1.8)$ \\
Total & 510 & $106(20.8)$ & $13(2.6)$ & $6(1.2)$ & $20(3.9)$ \\
\hline
\end{tabular}

Values are presented as number (\%).

a) $\mathrm{P}=0.042,{ }^{\text {b) }} \mathrm{P}=0.125$.

ROSC, return of spontaneous circulation; $\mathrm{PCl}$, percutaneous coronary intervention; MTH, mild therapeutic hypothermia.

\section{Multivariate analysis for survival to discharge}

In the multivariate logistic regression analysis, victim's age, location of arrest, witness of arrest and initial rhythm were significantly correlated with survival to discharge (Table 5). Patient gender, bystander CPR, and time between emergency call and EMS arrival were not statistically significant.

\section{DISCUSSION}

Despite numerous large-scale training efforts and multiple iterations of guidelines, OHCA remains a leading cause of death and a major public health problem in the industrialized world. ${ }^{1-3}$ Survival is very low in most jurisdictions, and there are large variations in survival rates. Such variability is presumably related to the extent to which important interventions are implemented; immediate recognition of cardiac arrest, early CPR with an emphasis on chest compressions, rapid defibrillation, effective advanced life support, and integrated postcardiac arrest care are all believed to improve survival rates. ${ }^{14,6,10}$ The American Heart Association refers to these aspects of the OHCA response as the chain of survival. ${ }^{6}$ Strengthening this chain requires setting and meeting standards for implementing these interventions in EMS systems, hospitals, and the public. ${ }^{6,7}$

In Korea, various efforts have been made to determine OHCA incidence and survival rates. ${ }^{5,11}$ To our knowledge, however, this is the first study to report on the incidence, processes of care, and outcomes from OHCA in a specific regional community in Korea using the standardized Utstein template. ${ }^{9}$ Accurate and on-going measurement is believed to be a fundamental component of any system of resuscitation care. ${ }^{6}$ It is essential, therefore, to have not only national but also regional OHCA data in order to measure and track incidence, individual interventions, and the overall effectiveness of response systems. While we found the overall OHCA survival rate in Ansan City to be very low (4\%, with 6/778 [0.8\%] having a good neurological outcome), our results are similar to those previously published in Korea and significantly lower than
Table 5. The multivariate logistic regression model for survival to discharge

\begin{tabular}{lcccc}
\hline Characteristic & Adjusted $\mathrm{OR}^{*}$ & \multicolumn{1}{c}{$95 \% \mathrm{Cl}^{*}$} & P-value \\
\hline Gender & & & & \\
$\quad$ Male & 1.00 & & & \\
$\quad$ Female & 1.16 & 0.39 & 3.45 & 0.788 \\
Age (yr) & 0.94 & 0.91 & 0.97 & $<0.001$ \\
Location of arrest & & & & \\
$\quad \begin{array}{l}\text { Private home } \\
\text { Public place }\end{array}$ & 1.00 & & & \\
Arrest witnessed & 3.64 & 1.40 & 9.45 & 0.008 \\
$\quad$ No & & & & \\
$\quad$ Yes & 1.00 & & & \\
Bystander CPR provided & 3.58 & 1.35 & 9.50 & 0.010 \\
$\quad$ No & 1.00 & & & \\
$\quad$ Yes & 0.93 & 0.30 & 2.91 & 0.902 \\
Initial rhythm & & & & \\
$\quad \begin{array}{l}\text { Non-shockable } \\
\text { Shockable }\end{array}$ & 1.00 & & & \\
Call to EMS arrival time & 4.12 & 1.61 & 10.52 & 0.003 \\
$\quad 7$ min or less & & & & \\
\hline
\end{tabular}

${ }^{*}$ Odds ratio (OR) and 95\% confidence interval (Cl) was calculated with adjustment for related factors (gender, age, location of arrest, witness, bystander cardiopulmonary resuscitation [CPR], initial rhythm, and time between emergency call and emergency medical service [EMS] arrival).

results found in many reports from Europe, North America, and Japan. ${ }^{1,4,10}$ We believe that while the survival rates we found were low, this report is a key step in the process of improving outcomes from OHCAs in Ansan City. Accurately measuring incidence, processes, and outcomes will guide the standards of care by showing whether specific interventions are improving, having no impact or even lowering survival rates.

The chain of survival, composed of early recognition and EMS activation, early bystander CPR, rapid defibrillation, effective advanced life support and integrated postcardiac arrest care is important for enhancing the OHCA survival rate. ${ }^{6}$ Our study suggests that in Ansan City there are several links in the chain of survival that need to be strengthened. Of the 778 adult OHCA cases of presumed cardiac etiology, 295/778 (37.9\%) were witnessed by first responders, and this result was not significantly different from that of previous studies. ${ }^{4}, 10$ Nevertheless, only 103/778 (13.2\%) received bystander $\mathrm{CPR}$, a rate significantly lower than those reported in other studies. ${ }^{4,10-13}$ Considering the large impact bystander CPR has on survival, ${ }^{12-15}$ this deficit likely contributes to Ansan City's very low survival rate. One of our strategies going forward will be to target large-scale public and dispatch CPR education, training, and outreach aimed at improving bystander CPR. Of the 103 cases in which patients received bystander CPR, CPR instructions were given by dispatchers in 57/103 cases (55.3\%). As shown in Table 2, when lay rescuers were provided with CPR instructions by dispatch personnel, the rate of bystander CPR was significantly 
higher than the rate without instructions ( $51.4 \%$ vs. $6.9 \%, \mathrm{P}<$ 0.001). This finding suggests that dispatcher guidance is effective in increasing the incidence of prearrival CPR. Prearrival CPR instruction, in fact, has been suggested as a cost-effective strategy for increasing bystander CPR rates and OHCA survival. ${ }^{16-18}$ Of the 778 OHCA cases in our study, EMS personnel provided CPR instructions only in 111 cases (14.3\%). This finding reveals a clear opportunity for system improvement, especially since Ansan City has no standardized protocol for dispatcher-guided bystander CPR instruction. Another important strategy, then, will be to implement a 1-1-9 training program that focuses on instructing dispatchers to recognize cardiac arrest and instruct callers on how to perform chest compressions.

Rapid defibrillation has been shown to improve the likelihood of good outcomes following OHCA. ${ }^{6,7}$ In our study, the rate of trained EMS rescuer defibrillation for patients who required it was only $27.1 \%$. Our data suggest that EMS providers should receive more complete training with an emphasis on identification of ventricular dysrhythmias and the need for immediate defibrillation.

In its 2010 guidelines, the American Heart Association added integrated postcardiac arrest care to the chain of survival. ${ }^{6} \mathrm{Ap}-$ propriate postcardiac arrest care, including, when indicated, mild therapeutic hypothermia or percutaneous coronary intervention, is believed to be critical for obtaining good outcomes from OHCA. ${ }^{19-21}$ Our results showed that mild therapeutic hypothermia and coronary angiocardiography were performed infrequently during in-hospital postcardiac arrest care in the 3 medical institutions that treated more than 100 OHCA patients during the study period. While Ansan City hospitals already have standard guidelines for in-hospital postcardiac arrest care, our findings indicate that these are not being followed. Emergency care facilities in Ansan city need prompt education and continuing assessment and feedback to reinforce the importance of these postarrest procedures. Of the 12 medical institutions in Ansan City that accept cardiac arrest patients, 7 received fewer than 50 cardiac arrest patients in 4 years. Considering the human resources, equipment and facilities available at these low-volume institutions, integrated postcardiac arrest care may require the creation of a regional community plan for patient transportation after ROSC. $^{22,23}$

This study has several limitations. We retrospectively reviewed all of the available EMS and hospital data using the Utstein style of reporting, but it is possible that our data capture was incomplete, and there may have been some ascertainment bias with regard to the determination of arrest etiology. It is also possible that EMS and hospital databases contained erroneous informa- tion. Differences in the style and integrity of medical records among the 12 hospitals studied might have affected the accuracy of hospital data collection. Furthermore, our study of the survival group was limited, as the total number of patients and the number of patients who survived until discharge was small. More standardized electronic data collection may, in the future, help overcome such limitations.

In summary, our analysis of the incidence, processes of care and outcomes of OHCA in Ansan City, Korea, showed its survival rate to be very low. This is likely related to multiple variables within the chain of survival, several of which are modifiable. Our results identified key areas for improvement, a first step necessary in increasing OHCA survival in Ansan City. Our findings should prompt the Ansan City medical and public policy communities to join together, creating a comprehensive plan for an effective, integrated resuscitation system that will strengthen the OHCA chain of survival in Ansan City.

\section{CONFLICT OF INTEREST}

No potential conflict of interest relevant to this article was reported.

\section{ACKNOWLEDGMENTS}

The authors appreciate all emergency medical services providers in Ansan Fire Department, who dedicate themselves in spite of a harsh work environment. Also the sincere cooperation of emergency medical facilities and leadership from Public Health Center in Ansan City made this meaningful work possible.

\section{REFERENCES}

1. Berdowski J, Berg RA, Tijssen JG, Koster RW. Global incidences of out-of-hospital cardiac arrest and survival rates: systematic review of 67 prospective studies. Resuscitation 2010;81: 1479-87.

2. Bobrow BJ, Vadeboncoeur TF, Clark L, Chikani V. Establishing Arizona's statewide cardiac arrest reporting and educational network. Prehosp Emerg Care 2008;12:381-7.

3. Rea TD, Eisenberg MS, Sinibaldi G, White RD. Incidence of EMS-treated out-of-hospital cardiac arrest in the United States. Resuscitation 2004;63:17-24.

4. Nichol G, Thomas E, Callaway CW, et al. Regional variation in out-of-hospital cardiac arrest incidence and outcome. JAMA 2008;300:1423-31.

5. Ahn KO, Shin SD, Suh GJ, et al. Epidemiology and outcomes 
from non-traumatic out-of-hospital cardiac arrest in Korea: a nationwide observational study. Resuscitation 2010;81:974-81.

6. Travers AH, Rea TD, Bobrow BJ, et al. Part 4: CPR overview: 2010 American heart association guidelines for cardiopulmonary resuscitation and emergency cardiovascular care. Circulation 2010;122:S676-84.

7. Rea TD, Page RL. Community approaches to improve resuscitation after out-of-hospital sudden cardiac arrest. Circulation 2010;121:1134-40.

8. Ansan City. Ansan City statistical information system [Internet]. Ansan: Ansan City Holl: [cited 2013 Sep 10]. Available from http://stat.iansan.net/Main.jsp.

9. Jacobs I, Nadkarni V, the ILCOR task force on cardiac arrest and cardiopulmonary resuscitation outcomes. Cardiac arrest and cardiopulmonary resuscitation outcome reports. Update and simplification of the Utstein templates for resuscitation registries. Circulation 2004;110:3385-97.

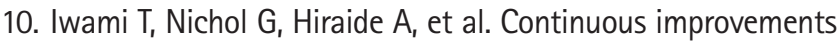
in "chain of survival" increased survival after out-of-hospital cardiac arrests: a large-scale population-based study. Circulation 2009;119:728-34.

11. Lee MJ. Incidence and outcome of cardiac arrest in Korea. J Korean Soc Emerg Med 2012;23:168-80.

12. Vadeboncoeur T, Bobrow BJ, Clark $L$, et al. The Save Hearts in Arizona Registry and Education (SHARE) program: who is performing CPR and where are they doing it? Resuscitation 2007;75:68-75.

13. Bobrow BJ, Spaite DW, Berg RA, et al. Chest compression-only CPR by lay rescuers and survival from out-of-hospital cardiac arrest. JAMA 2010;304:1447-54.
14. Holmberg M, Holmberg S, Herlitz J. Effect of bystander cardiopulmonary resuscitation in out-of-hospital cardiac arrest patients in Sweden. Resuscitation 2000;47:59-70.

15. Swor RA, Jackson RE, Cynar M, et al. Bystander CPR, ventricular fibrillation, and survival in witnessed, unmonitored out-ofhospital cardiac arrest. Ann Emerg Med 1995;25:780-4.

16. White L, Rogers J, Bloomingdale $M$, et al. Dispatcher-assisted cardiopulmonary resuscitation: risks for patients not in cardiac arrest. Circulation 2010;121:91-7.

17. Bradley SM, Rea TD. Improving bystander cardiopulmonary resuscitation. Curr Opin Crit Care 2011;17:219-24.

18. Bohm K, Rosenqvist M, Hollenberg J, Biber B, Engerstrom L, Svensson L. Dispatcher-assisted telephone-guided cardiopulmonary resuscitation: an underused lifesaving system. Eur J Emerg Med 2007;14:256-9.

19. Peberdy MA, Callaway CW, Neumar RW, et al. Part 9: postcardiac arrest care: 2010 American heart association guidelines for cardiopulmonary resuscitation and emergency cardiovascular care. Circulation 2010;122:S768-86.

20. Hypothermia after Cardiac Arrest Study Group. Mild therapeutic hypothermia to improve the neurologic outcome after cardiac arrest. N Engl J Med 2002;346:549-56.

21. Bernard SA, Gray TW, Buist MD, et al. Treatment of comatose survivors of out-of-hospital cardiac arrest with induced hypothermia. N Engl J Med 2002;346:557-63.

22. Bobrow BJ, Kern KB. Regionalization of postcardiac arrest care. Curr Opin Crit Care 2009;15:221-7.

23. Heffner AC, Pearson DA, Nussbaum ML, Jones AE. Regionalization of post-cardiac arrest care: implementation of a cardiac resuscitation center. Am Heart J 2012;164:493-501. 